\title{
Nebulised antibiotherapy: conventional versus nanotechnology- based approaches, is targeting at a nano scale a difficult subject?
}

\author{
Esther de Pablo ${ }^{1}$, Raquel Fernández-García ${ }^{1}$, María Paloma Ballesteros ${ }^{1,2}$, Juan José Torrado ${ }^{1,2}$, Dolores R. \\ Serrano $^{1,2}$ \\ ${ }^{1}$ Departamento de Farmacia y Tecnología Farmacéutica, Facultad de Farmacia, Universidad Complutense de Madrid, Plaza Ramón y Cajal s/ \\ n, Madrid, Spain; ${ }^{2}$ Instituto Universitario de Farmacia Industrial (IUFI), Facultad de Farmacia, Universidad Complutense de Madrid, Avenida \\ Complutense, Madrid, Spain \\ Contributions: (I) Conception and design: E de Pablo; (II) Administrative support: None; (III) Provision of study materials or patients: None; (IV) \\ Collection and assembly of data: None; (V) Data analysis and interpretation: None; (VI) Manuscript writing: All authors; (VII) Final approval of \\ manuscript: All authors. \\ Correspondence to: Dolores R. Serrano. Departamento de Farmacia y Tecnología Farmacéutica, Facultad de Farmacia, Universidad Complutense de \\ Madrid, Plaza Ramón y Cajal s/n, Madrid 28040, Spain. Email: drserran@ucm.es.
}

\begin{abstract}
Nebulised antibiotics offer great advantages over intravenously administered antibiotics and other conventional antibiotic formulations. However, their use is not widely standardized in the current clinical practice. This is the consequence of large variability in the performance of nebulisers, patient compliance and a deficiency of robust preclinical and clinical data. Nebulised antibiotherapy may play a significant role in future pulmonary drug delivery treatments as it offers the potential to achieve both a high local drug concentration and a lower systemic toxicity. In this review, the physicochemical parameters required for optimal deposition to the lung in addition to the main characteristics of currently available formulations and nebuliser types are discussed. Particular attention will be focused on emerging nanotechnology based approaches which are revolutionizing inhaled therapies used to treat both infections and lung cancer. Promising carriers such as Trojan-Horse microparticles, liposomes, polymeric and lipid nanoparticulate systems have been investigated and proposed as viable options. In order to achieve sitespecific targeting and to optimize the PK/PD balance critical nanoscale design parameters such as particle size, morphology, composition, rigidity and surface chemistry architecture must be controlled. Development of novel excipients to manufacture these nanomedicines and assessment of their toxicity is also a keystone and will be discussed in this review.
\end{abstract}

Keywords: Pulmonary infections; particle size; pulmonary deposition; nebulisers; Trojan Horse microparticles; nanoparticles; antibiotherapy

Submitted Aug 10, 2017. Accepted for publication Sep 08, 2017.

doi: $10.21037 /$ atm.2017.09.17

View this article at: http://dx.doi.org/10.21037/atm.2017.09.17

\section{History of nebulised drugs}

One of the first described inhalation therapies, was the use of atropine cigarettes obtained from the leaves of the atropa belladonna plant, for treating respiratory ailments occurring in the chest and throat. Limited reports of cigarettes and inhalation vapors appeared in medical literature during the following centuries. One of the most remarkable publications was the use of "Potters Asthma Cigarettes" (with datura stramonium leaves) and its bronchodilator action similar to that of ipratropium aerosol. In the early nineteenth century, the steam driven device appeared as the first inhalation therapy. Even though, several nebulizing devices were developed for therapeutic and diagnostic purposes, the current use of nebulised drugs remains in third position behind metered dose inhalers (MDIs) and dry 


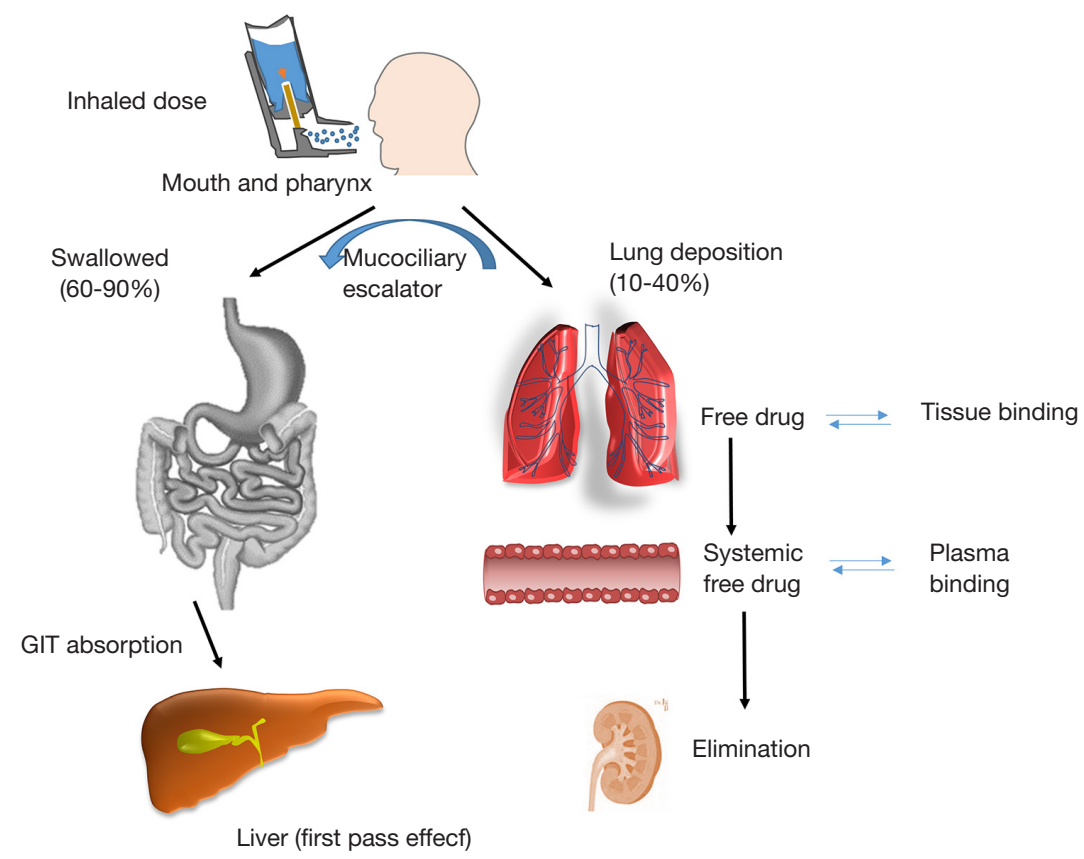

Figure 1 Fate of an inhaled active pharmaceutical ingredient (API): pharmacokinetic/pharmacodynamic (PK/PD) profile. Modified from: $(3,4)$.

powdered inhalers (DPIs). However, nebulisers can offer advantages over both MDIs and DPIs, for example through the ability to use existing intravenous drug solutions off license. Other notable advantages of aerosols or nebulised products are their ease of use, particularly amongst geriatric and paediatric populations as well as their suitability for emergency treatments and the capability to deliver higher drug amounts when compared with either DPIs or MDIs (1-3).

\section{Pulmonary drug delivery}

\section{Aerosol deposition and airway physiology}

Developing an efficient nebulised therapy requires a deeper understanding of the airway physiological pathway, aerosol deposition and physico-chemical properties of the drug product (Figure 1). The first question to be addressed is how much and where the aerosol particles are deposited within the lung cavity. Another key point is to know how long the drug will remain in the lung as this will dictate the posology and dose to be administered which will depend on (I) physicochemical properties of the active ingredient (e.g., dissolution rate, mucociliary transport and tissue binding); and (II) free drug concentration present in the lung which correlates with the pulmonary effect. It is also important to note that on average $60-90 \%$ of all aerosol particles will be swallowed and thus, it is critical to know the oral drug bioavailability, plasma protein binding levels, elimination rates as well as the systemic correlation between the free drug concentration and adverse effects. In those cases where the drug administered is a prodrug, it is necessary to know how efficient is the activation process within the lung and also, the activation in the bloodstream for those swallowed particles $(5,6)$.

It is possible to predict how the formulation will behave after pulmonary administration by accounting for mechanisms of aerosol deposition in the lung tissue and the factors that may affect the aforementioned deposition. Among these factors, the air velocities within the respiratory tract is of vital importance as well as the different diameters and angles of branching of the anatomic regions and the breathing cycle.

After nebulization, the drug passes through many physical and physiological barriers consisting of multiple bifurcations and the immune system (1). A thick surfactant layer is secreted by ciliated columnar epithelium cells located in the upper airway with the aim of protecting the lungs by removing foreign particles through the mucociliary escalator system (5).

Particles deposit in the respiratory track depending on their size, morphology and surface chemistry. The 


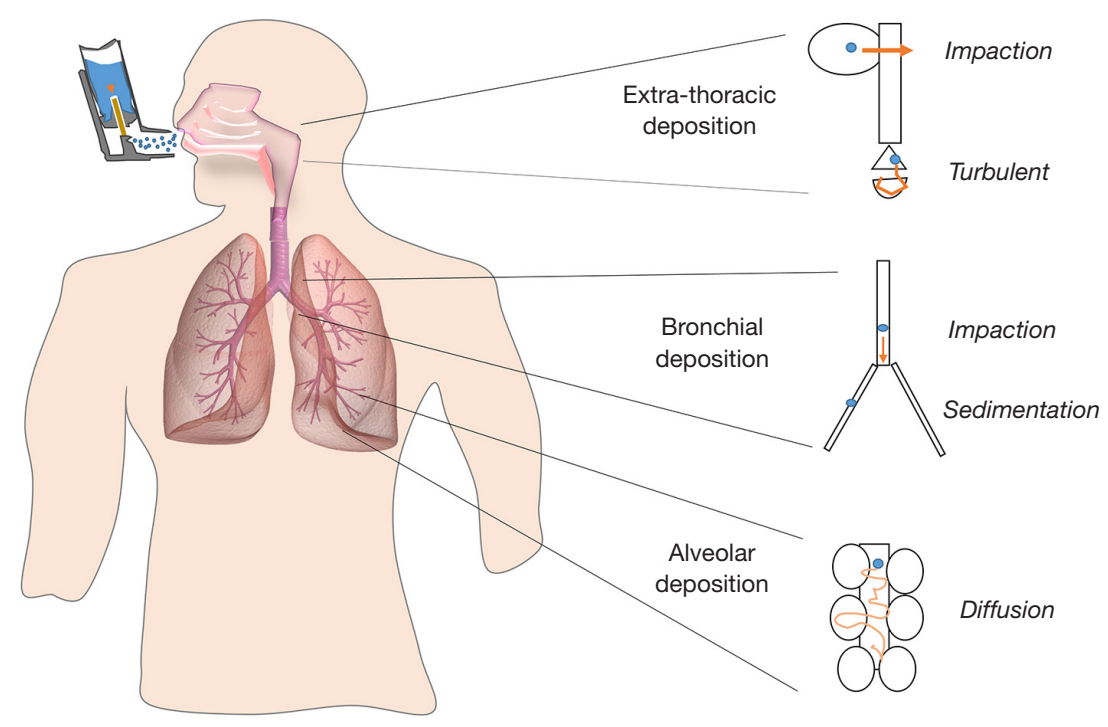

Figure 2 Deposition pattern of inhaled particles within the upper, mid and lower respiratory tract. Modified from: $(3,7)$.

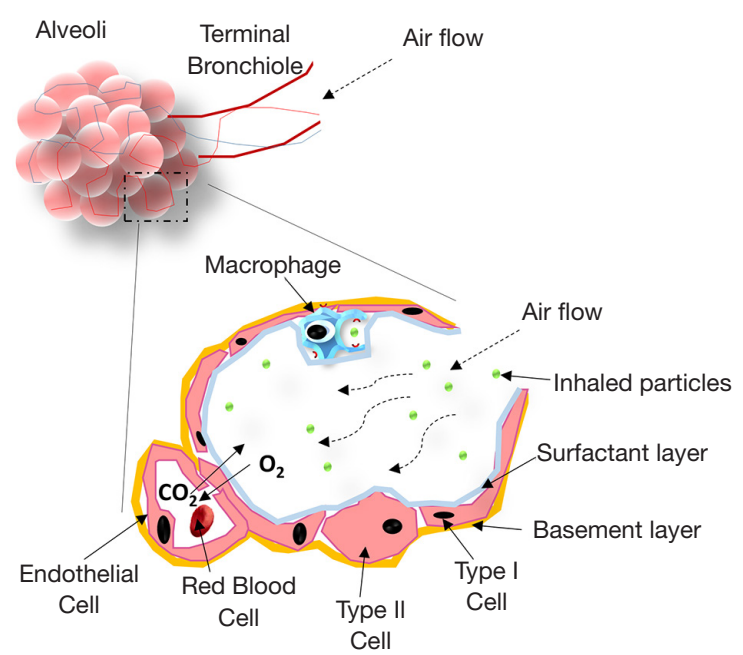

Figure 3 Diagram of the alveoli and inhaled particle deposition in the respiratory tract. Modified from: $(8,9)$.

three main mechanisms of particle deposition are: (I) impaction; (II) sedimentation; and (III) diffusion (Figure 2). As inhaled particles travel down the respiratory tract, the airways become narrower, but at the same time more numerous increasing the cross-sectional area of the airways and reducing the air flow velocity. In the upper part of the respiratory tract, the speed of inhaled air is largest in the central airways and those particles larger than $5 \mu \mathrm{m}$ (Mass Median Aerodynamic Diameter, MMAD) will be removed mainly by impaction. In the mid and lower tract (smaller bronchi, bronchioles and alveoli), particles will be removed by a combination of impaction, sedimentation and diffusion mechanisms. When the particles possess a smaller size than $5 \mu \mathrm{m}$, the chance of peripheral penetration and retention increases. The optimal MMAD for lung deposition is considered to be between 1 to $5 \mu \mathrm{m}$. However, for very fine particles $(<0.5 \mu \mathrm{m} M M A D)$, there is increasing probability of not being deposited in the lung parenchyma and be subsequently exhaled $(1,4)$.

A final line of protection within the lung tissue is provided by alveolar macrophages which can phagocyte particles reaching the deep alveolar region specially those between 1 and $2 \mu \mathrm{m}$ (Figure 3) (8). To evade both the clearance by macrophages and the mucociliary trapping and clearance, the inhaled particle should be small enough to pass across the upper part and possess a suitable surface chemistry to avoid adhesion to the mucosal layer. The main advantage when treating lung infections is that mucociliary escalator system is compromised facilitating the penetration through the mucus and hence the drug efficacy (10). For example, certain pharmaceutical technological approaches such as PEGylation facilitate the penetration across the mucus layer (5). The entrapment and removal of particles from the mucus layer usually occurs through hydrophobic interactions and formation of polyvalent adhesive interactions due to the negative charges of the mucin proteoglycans (11). Polyethylene glycol (PEG) possesses a near-neutral surface charge with negligible protein 
adsorption and hence, PEG coated particles have shown an enhanced transport across mucus layers $(11,12)$.

\section{Formulation and nebuliser performance}

In nebulisers, the drug is commonly formulated as either a suspension or an aqueous solution, which is atomized into fine droplets via an external nebulization source during the inhalation process (1). Sterility of these aqueous-based inhalation solutions and suspensions must be ensured and to achieve this, formulations are usually packaged in single-use containers $(1-3 \mathrm{~mL})$. Sterile water for injection is normally used as solvent, and in some cases, ethanol or propylen glycol can be employed as co-solvents.

Historically, the off license use of intravenous drug solutions as aerosols was common, however, it had many drawbacks as the formulations were not originally intended for lung delivery such as unpleasant taste, irritation or bronchoconstriction (9). Nowadays, although the development of inhaled formulations has reduced the risk of adverse effects, formulation scientists have to carefully consider the necessity of including certain excipients in the formulation in order to improve the physicochemical properties of drugs as the potential for adverse effects always exists. For example, antimicrobial preservatives such as benzalkonium chloride or parabens are usually needed but they can cause allergic reactions (13).

Physical stability of suspensions is vital to avoid aggregation of particles. Surfactants such as polysorbates and sorbitans are commonly added to facilitate the dispersion or dissolution of drugs. Interaction with packing components can occur and hence compatibility assays should be performed in particular with proteins and peptides that have high tendency to adsorb onto plastic materials. The addition of antioxidants like ascorbic acid or metabisulphites and chelating agents like EDTA may be required when the influence of oxygen, light and trace metals lead to drug degradation $(1,14)$.

Other critical factors to note when developing inhaled formulations are: $\log \mathrm{P}, \mathrm{pKa}$, isoelectric point and solubility. Tonicity and solution $\mathrm{pH}$ tend to be a problem during preformulation as acidic $\mathrm{pH}$ and non-isotonic aerosols can provoke bronchoconstriction which is of major importance in patients with asthma (4). The lungs have limited buffering capacity unlike the blood and thus, isotonicity and $\mathrm{pH}$ must be adjusted (targeting neutral solutions of $300 \mathrm{mOsm} / \mathrm{L}$ ) in order to avoid bronchoconstriction by the addition of $\mathrm{pH}$ and osmolarity regulators such as $\mathrm{HCl}$,
$\mathrm{NaOH}$, citric acid, phosphates, trometamol and/or $\mathrm{NaCl}$ and other salts (15).

Regarding nebuliser performance, the critical quality attributes (also denominated as CQAs) of the product need to be assessed and maintained across of all supported devices. Amongst them, drug delivery rate (output) and aerodynamic droplet size distribution are usually the most relevant factors. It is important to study in depth and understand how the key formulation parameters, such as viscosity, surface tension and drug concentration, influence these CQAs. An increase in solution viscosity could slow down the nebulization and reduce the drug output for jet nebulisers but also lead to decreased particle size, and modification of the drug deposition pattern $(16,17)$. The electrostatic charge in the aqueous solutions can inhibit the flow and detachment of fluid through the mesh, and the introduction of electrolytes can suppress the charges improving particle size, drug output and nebulization time. Drug concentration levels have been known to have a negative effect on nebuliser output but this has been demonstrated to be specific to certain nebuliser types $(6,18)$.

\section{Types of nebulisers and methods of nebulisation}

The main principle of all drug nebulisers is based on the fact that an aqueous drug solution or suspension is transformed into an aerosol containing the drug dispersed in fine droplets which will be consequently inhaled and deposited in the lung parenchyma. By controlling the rate (or amount) of drug aerosol and its particle size, a desired dose of drug can be delivered and site-specific targeted within the lung cavity, thereby minimising dosage and systemic distribution as well as side effects.

However, significant differences in aerosol output and particle size exist among the different nebuliser devices. Even with the same nebuliser, different droplet size distribution can take place when using different compressors or pressures. It is important to note that one cannot select a theoretical nebuliser design suitable for all applications. It is not possible for a single device to be employed where individual patient needs, target areas and desired doses must be accommodated. For example, inspiratory flow rate and lung volume capacity are significantly altered in patients with pulmonary infections or chronic diseases such as asthma and chronic obstructive pulmonary disease (COPD) affecting drug output and deposition (19). In order to improve delivery efficiency, some breath devices such Pari LC Star or AeroEclipse II have been created in such a way 
that aerosols are generated only during inspiration (20).

Regarding the method for aerosol generation, it is key to use systems that keep constant the particle size distribution and the drug concentration in the solution or suspension. These parameters are highly affected for the method of aerosolisation and the duration of the process and will significantly affect the characteristics of the resulting aerosol. Particles between 0.5 to $5 \mu \mathrm{m}$ are the ones that can reach the lung and the pulmonary alveoli giving a local effect during the administration of the drug.

Larger particles will be eliminated by impaction not reaching the mid and deeper lung parenchyma and smaller particles will be exhaled, without achieving efficacy in any of the cases. The generation of the highest amount of particles or droplets within this range is necessary for the successful delivery of the drug.

The most commonly utilized systems for pulmonary delivery are the following: (I) the evaporation-condensation method which is not very stable and reproducible and generates a result with particle sizes from 0.1 to several microns; (II) electrospray which is based on electrostatic charges that decreases the surface tension and cause breakup of the surface of the liquid; (III) nebulisers that employ either compressed air or ultrasounds for air blast atomization of liquids; and (IV) spinning-disk based on the breakup into larger and smaller droplets created at the edges of the rotating disk (5). One of the major drawbacks associated with the electrospray method is the generation of small particles between 0.002 and $0.3 \mu \mathrm{m}$, which are easily exhaled resulting in less efficacy. On the contrary, the spinning disk system leads to the formation of larger particles (up to $100 \mu \mathrm{m}$ ) compared to other techniques that will not reach the lung. Overall, nebulisers generate aerosols with the best particle size characteristics between $0.01-5 \mu \mathrm{m}(3)$.

The two classical devices for nebulization are: jet and ultrasonic nebulisers which operate using compressed air and vibrating piezo-electric crystals respectively $(18,21)$. Jet nebulisers are still the most utilised device for inhalation of solutions and suspensions. However, these systems have some disadvantages such as noise, less comfortable to carry and long dosing duration (10-15 min). The cooling effect of jet nebulisers due to the expansion of atomising gas and evaporation of solvent also influences patient's use. In contrary, ultrasonic nebulisers are compact and silent, and the dosing time is shorter. Nevertheless, heat may often be generated during ultrasonic nebulization, making then unsuitable for heat sensitive drugs.
Vibrating mesh nebulisers, a new generation of nebulisers, are a recent technology that uses vibrating perforated mesh to generate respirable sized droplets. The main advantages of these nebulisers are: (I) fast treatment time; (II) minimal residual dose; and (III) reduced drug waste. Spiriva Respimat ${ }^{\circledR}$, commercialised by Boehringer Ingelheim, works by creating a slow-moving aerosol cloud within a pocket size form factor. Due to the inherent differences in terms of atomisation mechanisms and device designs, even though the device is still classified as nebuliser, many clinicians prefer to consider it as a propellant free MDI or classified it as a metered dose liquid inhaler (MDLI). This portable device format allows for multiple doses and enables drug delivery in a single breath of moistened vapour without the need for continuous inhalations and is seen as the future direction of new nebulisers (22).

Another marketed nebuliser with enhanced performance characteristics is the AERx ${ }^{\circledR}$ Pulmonary Drug Delivery System. In this system, a bolus of aerosol particles is generated at the same time that the inspiration process takes place (23). The AKITA $^{\circledR}$ Inhalation System (Activaero) is another advanced aerosol technology where the nebulisation is controlled by a computer programmed to mimic the lung function characteristics and the inhalation pattern of the patient (24).

\section{Nebulised therapies in clinical practice}

Currently nebulisers remain widely utilised in hospitals and home settings. The major advantage of the nebulisers is that allow patients to inhale drug aerosols with tidal breathing movements and little training required. Nebulisers are especially suitable for patients unable to coordinate their breathing or activate MDIs or DPIs, such as the geriatric and paediatric population. Nebulised therapy is primarily intended as a treatment for pulmonary infections or chronic diseases such as asthma and COPD (Tables 1,2) (3).

\section{Asthma and COPD}

Most currently marketed inhalation solutions and suspensions used to treat bronchospasm during asthma and COPD consist of one or two combined drugs which can behave as: (I) short acting 82 -agonist (SABA) like salbutamol; (II) long acting 82 -agonist (LABA), such as formoterol; (III) short-acting muscarinic antagonist (SAMA), such as ipratropium; and (IV) long-acting muscarinic antagonist (LAMA), like tiotropium. Several 


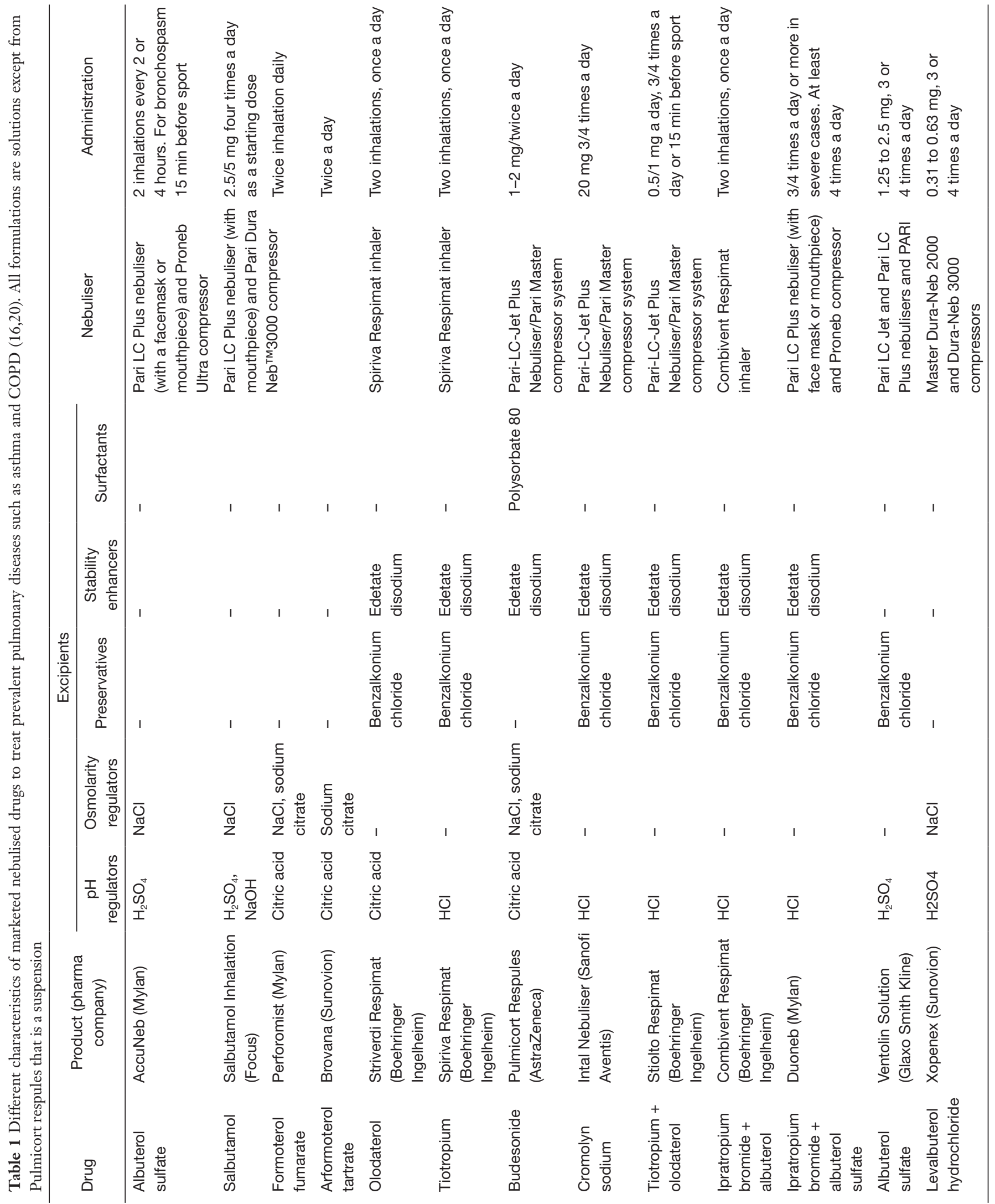




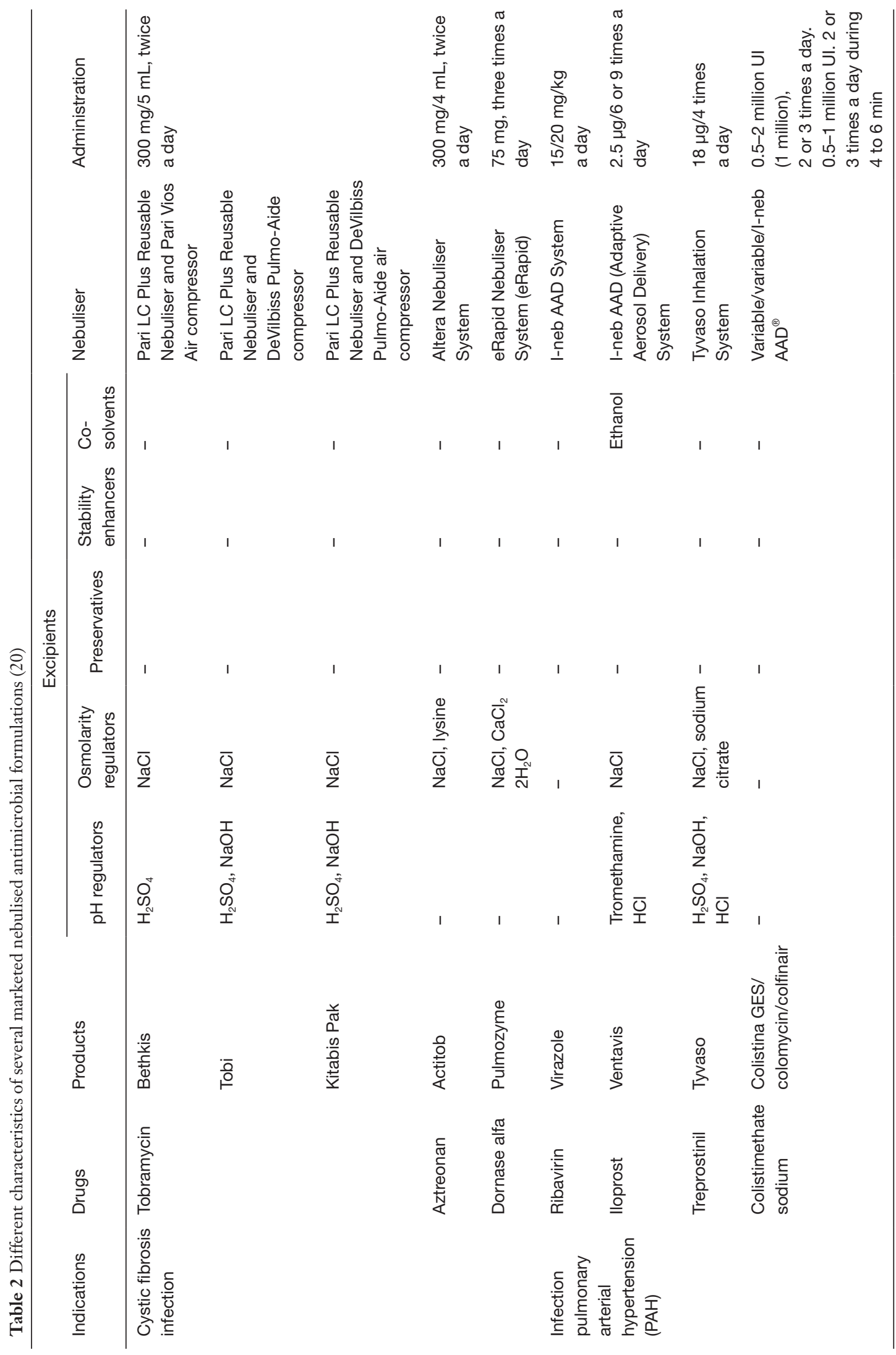


administrations per day are usually required to obtain the desired effect. However, the FDA has recently approved the inhalation spray of a LAMA/LABA combination (tiotropium/olodaterol), which can be delivered to patients as a single daily dose $(16,25)$. Inhaled corticosteroids such as budesonide are also common (Table 2). Most of the excipients employed are those described in section "Formulation and nebuliser performance" (Table 1).

\section{Pulmonary infections}

Nebulisers have the advantage that can continuously deliver drugs for a long dosing duration, being suitable for the administration of large doses, generally required to treat infections. Also, the administration of aerosol antibiotics through the pulmonary route allows high drug concentrations at the infection site (26). This may decrease intravenous toxicity, particularly the renal adverse effects, commonly caused by intravenous administration of aminoglycosides, colistin and amphotericin B (27).

Tobramycin was the first antibiotic solution approved for inhalation for the treatment an also the prevention of Pseudomonas aeruginosa infection in patients suffering from cystic fibrosis (CF). A long-term therapy study indicated that inhaled tobramycin improved lung function and reduced exacerbation rate for patients with CF (28). Multiple nebulised formulations are currently in the market to treat this disease (Table 2).

Inhalation antibiotic therapy has been also investigated for both prophylactic and acute treatment of Gram-negative and, fungal pneumonia. It has previously been shown that intermittent or short-term use of prophylactic inhaled antibiotics can be effective in decreasing colonisation of the airways by Gram-negative bacteria and reducing the incidences of pneumonia. However, increased bacterial resistance is a global health problem, and prolonged use of antimicrobials is associated with an unacceptably high prevalence of pneumonias caused by multi-drug resistant (MDR) bacteria. The use of aerosolised antibiotics should therefore be limited to well established bacterial pulmonary infections $(29,30)$.

The safety of nebulised antibiotherapy has been proven in numerous studies. Its efficacy as concomitant treatment along with parenteral drug administration or in monotherapy has been also demonstrated when treating respiratory infections in mechanically ventilated (MV) patients. Nebulised antibiotherapy has several advantages in this patient group such as: (I) improvement of pharmacokinetic profile compared to traditional systemic administration of antibiotics; (II) prevention of MDR bacteria; and (III) minimization of side effects and drug interactions. Until recently, development of this technique was slow, partly due to the limited knowledge of the determinants of aerosolisation during mechanical ventilation and poor technique implementation. The major disadvantage of conventional nebulisers was that lower amounts of drug reached the alveolar region due to great amounts of particles remained trapped either in the tracheobronchial tree or ventilator circuits. On the contrary, the latest generation of nebulisers has improved considerably the inhaled particle deposition and antibiotic aerosolisation in $\mathrm{MV}$ patients has received renewed interest (1).

However, multiple meta-analyses have failed to show a clear effect due to the heterogeneity of the studies. The optimal use of nebulised antibiotherapy can be achieved if techniques are standarised, indications are clarified and clinical guidelines are implemented. An important limitation in many studies has been the inadequate characterisation and control of variables affecting the efficiency of drug deposition, such as nebuliser design, operating conditions, particle size and patient's breathing pattern.

\section{Clinical trials on nebulised antibiotherapy}

Clinical trials on nebulised antibiotherapy have increased in recent years especially in the treatment of local diseases (Table 3). One such drugs that has awoken interest is amphotericin B probably because it is an antifungal drug with a broad spectrum of action and limited resistance but its adoption is limited in clinical practice due to its severe nephrotoxicity after intravenous administration $(32,33)$. The efficacy and safety of nebulised amphotericin B lipid complex (ABLC) and liposomal formulation (AmBiosme ${ }^{\circledR}$ ) are being evaluated in clinical trials in neutropenic hematooncologic patients for prophylactic use against invasive pulmonary aspergillosis. Preliminary data has shown promising results in reducing the incidence of pulmonary aspergillosis in this patient group (31). The use of other antibiotics such as tobramycin and gentamicin are also being investigated in clinical trials for pulmonary administration. All the formulations undergoing clinical trials are of extreme importance for clinicians as a guide about the use of new inhalable therapies as well as the prevalence of microbial contamination that may be associated with the use of inhalation devices. 


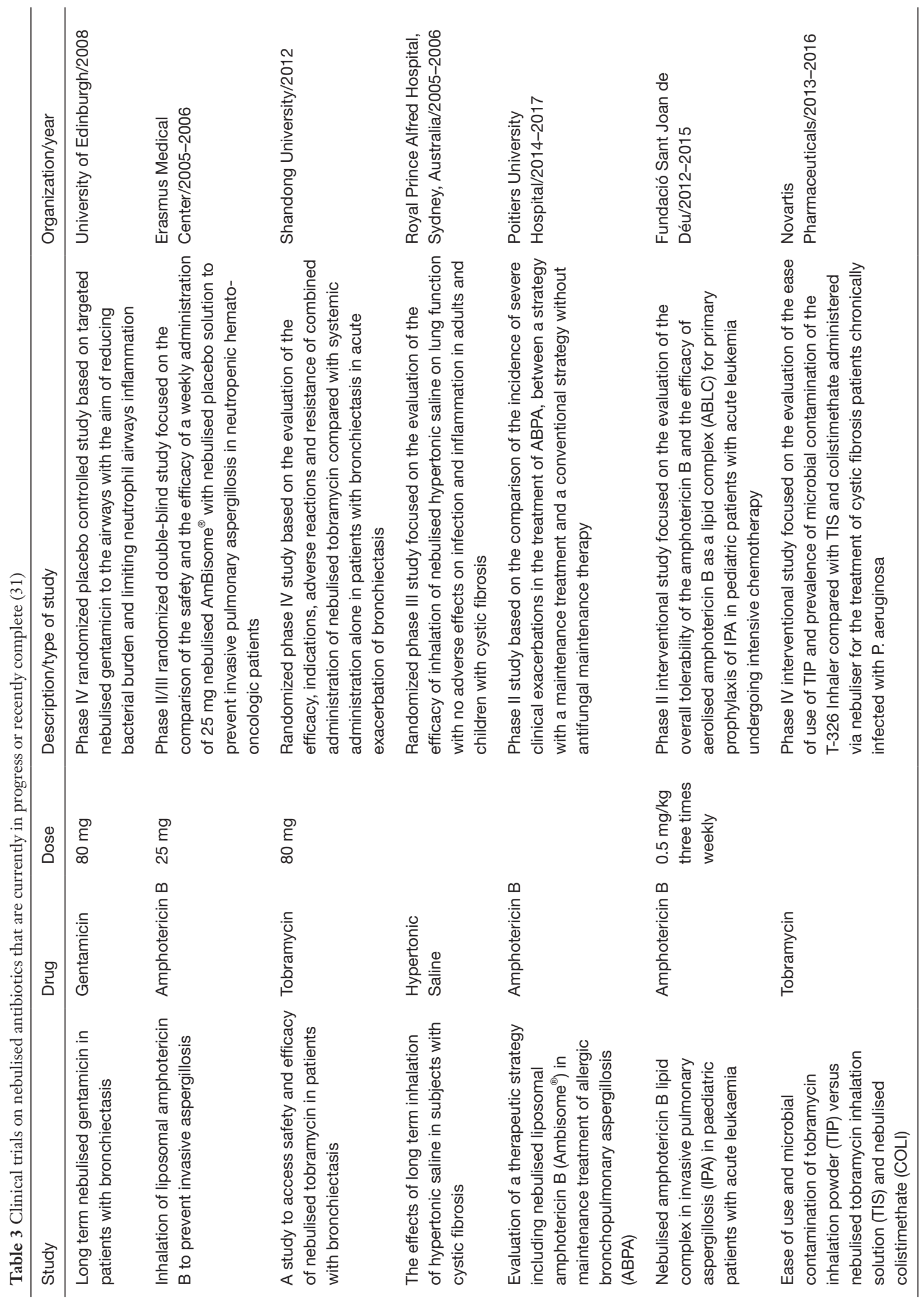




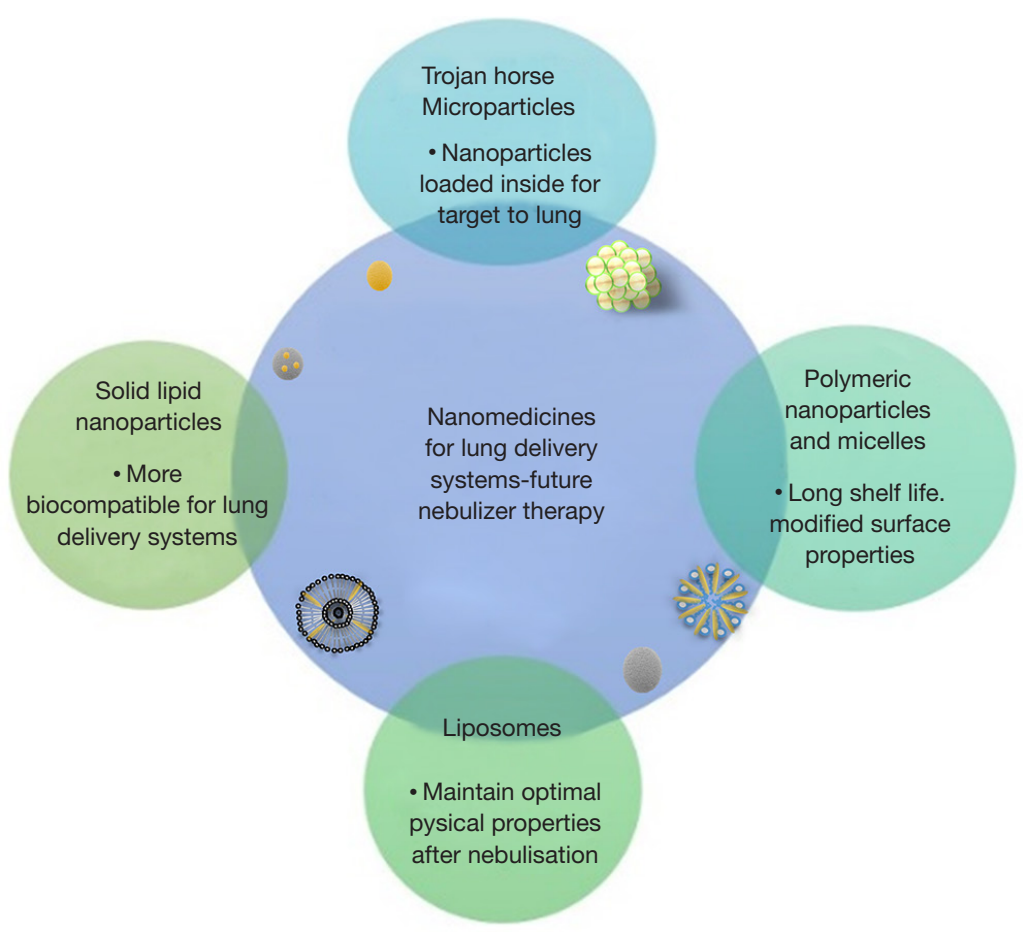

Figure 4 Nanomedicines characteristics of future lung delivery systems on nebulised therapy.

\section{Nano scale for lung delivery systems}

Pulmonary drug delivery has many advantages over other routes such as oral or parenteral administration. It is an attractive route due to two main reasons: (I) its non-invasive character allowing self-administration that leads to enhanced patient compliance and adherence to treatment; and (II) its large surface area for absorption along with reduced drug degradation and high permeability $(34,35)$. This route has been used successfully for local delivery of a variety of APIs including proteins, peptides, chemotherapeutics, antibiotics and vaccines that could not have been possible via other administration routes $(36,37)$.

In order to achieve successful pulmonary drug delivery, particle engineering is a key factor to take into account when developing inhalable formulations. Micro and nanoparticulate systems have been especially useful in sustained delivery and drug targeting with enhanced therapeutic effects (38).

An aerodynamic particle size below $5 \mu \mathrm{m}$ is crucial for particles reaching the mid and deep lung parenchyma. However, bearing in mind that particles below $0.5 \mu \mathrm{m}$ in size are easily exhaled, the development of successful nanotechnological approaches for lung application is not an easy task. Amongst all the critical nanoscale design parameters, particle size, morphology, composition, rigidity and the surface chemistry architecture have to be controlled in order to optimise the PK/PD balance and to achieve sitespecific targeting.

The combination of a variety of techniques at the micro and nanoscale has transformed pulmonary drug delivery. The most successful approaches will be discussed in the next section (Figure 4) (1).

\section{Trojan Horse microparticles}

One nanotechnology strategy for lung delivery is the use of "Trojan Horse microparticles" which are basically porous nanoparticles assembled within microparticulate systems (commonly between $2-5 \mu \mathrm{m}$ in diameter), in such a way that the potential for drug targeting of the nanoparticles and the better flow and aerolisation properties of the microparticles are combined in the same system.

Trojan Horse microparticles have been successfully produced using different strategies such as spray drying of solutions consisting of polymeric and nonpolymeric nanoparticles (39) or using supercritical $\mathrm{CO}_{2}$ as 
antisolvent (40). During the spray drying process, Van der Waals forces are created which keeps the nanoparticles together within a matrix of excipients such as phospholipids or other polymers. Upon inhalation, the microparticulate systems dissolve releasing the nanoparticles within the lung parenchyma.

As an example for the treatment of lung cancer or bacterial infection, superparamagnetic iron oxide Trojan Horse microparticles (MMAD of $2.2 \mu \mathrm{m}$ ) have been produced utilising PEG, hydroxypropyl-ß-cyclodextrin, ammonium carbonate and magnesium stearate as excipients. The particles are highly sensitive to the magnetic field and the targeting and deposition within the lung parenchyma can be facilitated by means of a magnet (41).

\section{Polymeric nanoparticles and micelles}

The use of multiple polymers for pulmonary drug delivery have been thoroughly investigated such as: (I) polycaprolactone; (II) alginate; (III) poly(lactic acid); (IV) poly(lactic-co-glycolic acid); (V) carbohydratebased polymer such as chitosan; and (VI) gelatin base $(42,43)$. The usage of these polymers has numerous advantages such as high drug encapsulation, sustained release, long shelf life, modified surface properties and prevention of drug degradation. Amongst all excipients for building nanoparticulate systems, block copolymers, chitosan and PEG are the most successful for achieving optimal sustained drug release and improved uptake (44). In addition, PEGylation also avoids phagocytosis of the particles and prolongs their halflife in the lung parenchyma (45). Nevertheless, in order to promote their biodegradability, modification of the polymer surface chemistry is required for therapeutic purposes and actually, their degradation rate in physiological media must be analysed along with toxicity assays to check the efficacy/toxicity balance of the carriers.

Multiple studies have been performed loading polymeric nanoparticles with anti-cancer drugs for targeted lung diseases. For instance, paclitaxel was loaded into polymeric particles consisting of PEG and polymer poly(ethylene oxide)-block-distearoyl phosphatidylethanolamine. The intratracheal instillation administration of the particles showed better drug absorption and improved drug localisation in the lung parenchyma in comparison with the intravenous administration of taxol (marketed formulation) (46).

\section{Solid lipid nanoparticles}

They are single layered solid particles consisting of solid lipid as matrix material, emulsifier and water which can be found physiologically. Several techniques have been successfully employed in the manufacture of these systems such as solvent evaporation or high pressure homogenization which are easily scalable from an industrial point of view. Good results in treating lung infections have been achieved when formulating amikacin with cholesterol using the latter strategy (47).

Phospholipids are found in most regions of the lung parenchyma at the alveolar surface (Figure 3). They are essential for friction reduction and maintaining of surface tension necessary for the proper functioning of the breathing mechanism $(42,48)$. These type of nanoparticulate systems have shown a better efficacy/toxicity ratio than the polymeric ones due to their composition based on physiological components that are less toxic and demonstrate greater biocompatibility towards pulmonary drug delivery. As an example, solid lipid nanoparticulate systems consisting of a 30:70 ratio of phospholipids and triglycerides has demonstrated a good safety profile after nebulisation with no activation of pro-inflammatory cytokines in mice (49).

\section{Liposomes}

The difference between solid lipid nanoparticles and liposomes is that the latter consists on single or multiple concentric lipid bilayers manufactured primarily from phospholipids and cholesterol encapsulating an aqueous environment. As solid lipid nanoparticulate systems, liposomes exhibit higher biocompatibility than polymeric nanoparticles and possess sustained release characteristics, enabling the maximum drug effect over a prolonged period of time. For example, AmBisome ${ }^{\circledR}$, amphotericin B-loaded liposomes are commonly used in the prophylaxis of pulmonary fungal infections; however, currently, it is only commercialised for parenteral use but yet not for pulmonary.

Nonetheless, inhaled liposomes are challenging to manufacture as it is critical to maintain their optimal physical properties after nebulisation (50). Currently, two such liposomal formulations are in the final stages of clinical trials prior to commercialisation as dry powder systems. Arikace $^{\circledR}$ (containing amikacin) and Pulmaquin ${ }^{\text {TM }}$ (containing ciprofloxacin) both developed for use in the treatment 


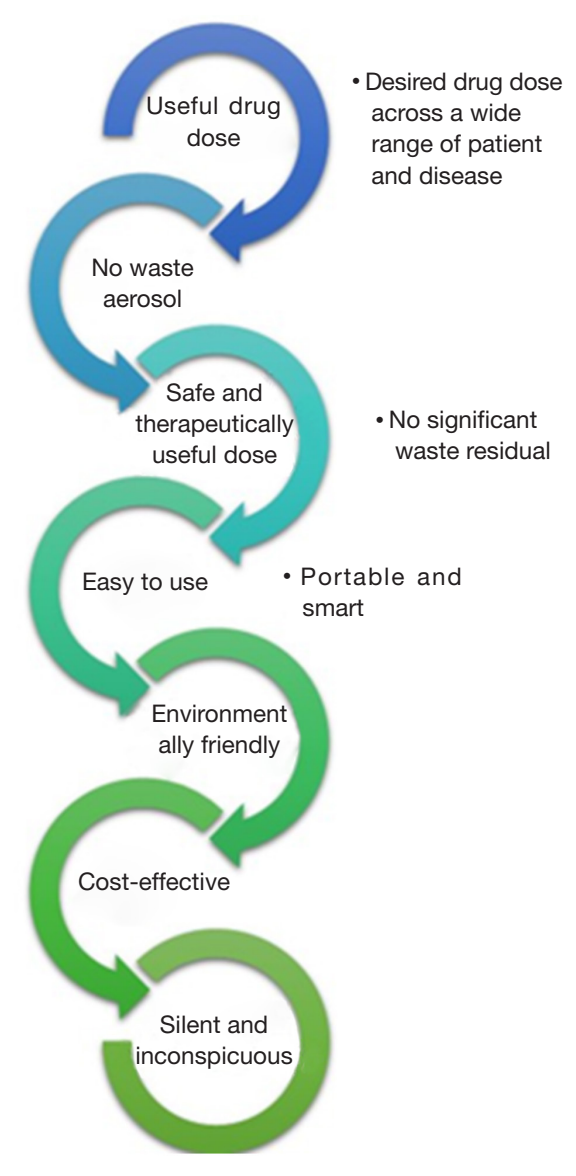

Figure 5 Characteristics of the ideal drug aerosol delivery system.

of pulmonary infections $(42,51)$. Arikace ${ }^{\circledR}$, consisting of amikacin, dipalmitoyl-phosphatidylcholine and cholesterol, is in phase 2 clinical trials with the aim of treating CFassociated lung infection caused by $P$. aeroginosa. After treatment with the liposomal inhaled formulation, an improvement in lung function and reduction in the number of colonies of $P$. aeruginosa in sputum was achieved along with a satisfactory safety and tolerability profile. A further application of liposomal formulations is the encapsulation of antioxidant molecules for the treatment of acute oxidantrelated lung injury (52).

\section{Regulatory perspective of inhalation products by EMA and FDA: bioequivalence and toxicity issues}

The European Standard recognizes that different nebulisers will deliver different doses of drug to the same patienteven if all conditions are controlled, such as breathing pattern, volume fill of drug solution or suspension and what is more, in a wide range of diseases and patient groups (3). Even though, a great effort is being doing in developing more effective and reproducible inhalation products, they are still far from ideal.

Ideally, inhalers should deliver the desired drug aerosol dose across a variety of drug preparations with no significant wasted residual drug left in the device after use. They should be easy to use for all patients including paediatric and geriatric populations. Nebulisers should be portable (small and light), silent, inconspicuous and smart (signals indicating when not functioning properly; dose delivery is complete; patient compliance monitor, etc.). They should be a cost-effective therapeutic device with limited wasted aerosol and environmentally friendly, with no damaging propellants such as chlorofluorocarbons and hydrofluoroalkanes and ideally recyclable. All these characteristics are summarised in Figure 5 (3).

The differences in bioequivalence studies of the inhalation devices and their high cost is an issue of concern for the regulatory bodies such as FDA and EMA. In 2009, the EMA issued the guideline focused on the demonstration of therapeutic equivalence between two inhaled products, based on a step-wise approach for the approval of generic inhaled formulations (53). From the point of view of the formulation, both the originator and the generic product should have an identical dosage form with the same API. Differences in crystalline structure, polymorphic form of the active ingredient or qualitative and quantitative variation in excipients should not unduly influence the pharmaceutical performance and efficacy/safety balance of the inhaled product.

The handling and resistance to airflow within the device for both the generic product and reference formulation should be the same. The CQAs to determine in vitro equivalence are: (I) dose delivery uniformity; and (II) particle size distribution (assessed by impactor stage), which should be within $15 \%$ of each other. The sole use of in vitro data is acceptable for approval when the generic formulation satisfies all of the above pharmaceutical criteria for equivalence. Otherwise, in vivo studies (pharmacokinetic or pharmacodynamics assays) may be required.

In 2013, five separate draft guidance were published by the FDA for each specific inhalation product: fluticasone propionate/salmeterol DPI and salbutamol sulphate, levalbuterol tartrate, ipratropium bromide, budesonide/ formoterol fumarate MDIs $(54,55)$. There are several challenges in gaining FDA approval for generic products 
as it requires that all tests including in vitro (single actuation content and MMDA), pharmacokinetic and pharmacodynamic assays demonstrate equivalence. In addition, in order to demonstrate the similarity of physical components for generic MDIs, plume geometry, spray pattern, priming and repriming studies may also be required.

The requirements for equivalence of DPI devices include: (I) premetered multi-dose format; (II) the device mechanism; (III) the dose administered; (IV) the operating procedure; (V) the size; (VI) the shape; (VII) the device resistance; and (VIII) the dose counter. It is also a requirement to demonstrate both qualitative and quantitative equivalence or sameness. Qualitative sameness (Q1) implies that the generic product uses the same inactive ingredient as the originator whereas the quantitative sameness (Q2) infers that the concentration of the inactive ingredients in the generic product must be within $5 \%$ of those in the originator. The guidance documents have been criticised and challenged by bodies representing the pharmaceutical industry and rather than follow the Q2 requirement, it has been proposed to incorporate a qualityby-design $(\mathrm{QbD})$ approach to study the control space of the excipient concentration for each generic product $(20,56,57)$.

It is worth noting that there are a limited amount of excipients approved for pulmonary applications and there remains a dearth of published literature guidance regarding safety and marketing authorization of pharmaceutical excipients for pulmonary use. Some guidelines mention the type of toxicity assays required to determine whether the use of a novel excipient is safe for human use (ICH, M3, S3A and S7A) (15). In order to achieve regulatory approval for a new excipient, a complete toxicological evaluation may be sufficient without to include an inhalation toxicology profile. Consequently, there remains no inhalation toxicology or LD50 data available for many well-known excipients (15).

Overall, pulmonary delivery is very challenging in terms of: (I) to show bioequivalence between innovator and generic inhaled formulations; and (II) to approve the use of nanoparticulate systems as inhaled therapies. One of the main reasons why there is no inhaled nanomedicines in the market is due to its potential toxicity, not only within the respiratory tract but also in extrapulmonary tissues that can be secondarily targeted. Currently, most drugs are very potent and many of them can produce adverse effects at the lung level specially in those patients with impaired pulmonary functions due to smoking and/or chemotherapy $(58,59)$. For example, inhalation of cytostatic drugs can have a significantly negative effect leading to severe complications on the lungs. However, this can be an advantage in treating lung cancer and targeting cytostatic agents towards the lung parenchyma, limiting penetration into the bloodstream and protecting the rest of the body from adverse effects $(35,42)$.

\section{Future perspectives and concluding remarks}

Nebulised antibiotics offer great advantages over intravenous administered antibiotics as well as other conventional antibiotic therapies. However, their use is not widely standardized in the current clinical practice leading to large variability between patients. This is the consequence of large differences in nebuliser performance and the lack of robust preclinical and clinical data. Nanotechnology based approaches are revolutionizing inhaled therapies especially in lung cancer and infections. Promising carriers such as Trojan Horses microparticles, polymeric and lipid nanoparticulate systems and liposomes are emerging. Amongst all the critical nanoscale design parameters, particle size, morphology, composition, rigidity and surface chemistry architecture have to be controlled in order to optimise the PK/PD balance as well as the efficacy/toxicity balance and to achieve sitespecific targeting. Development of novel excipients to manufacture these nanomedicines and assessment of their toxicity is also a keystone. Indeed, pharma companies need to evaluate carefully the benefits of using novel substance versus the delay in commercialisation due to objections from regulatory agents. For this reason, many companies prefer to use first, the few excipients already approved for inhalation in different concentrations and second, physiological and biocompatible excipients whose toxicity profile is well-known in other routes. In conclusion, the development of novel excipients and drug delivery systems is a current clinical need in order to achieve safer and more effective pulmonary formulations.

\section{Acknowledgements}

This work was supported by a grant from the Complutense University and Madrid Community Administration to the research group 91093.

\section{Footnote}

Conflicts of Interest: The authors have no conflicts of interest to declare. 


\section{References}

1. Poulakou G, Siakallis G, Tsiodras S, et al. Nebulized antibiotics in mechanically ventilated patients: roadmap and challenges. Expert Rev Anti Infect Ther 2017;15:211-29.

2. Lahzami S, Nicod LP. Inhaled therapies for cystic fibrosis. Rev Med Suisse 2011;7:2285-8.

3. Dennis JH, Pieron CA. Chapter 2: Quality control and standards in nebulizer performance and use. In: Boe J, O'Driscoll BR, Dennis JH. editors. Practical Handbook of nebulizer therapy. Ed. CRC Press, Taylor \& Francis Group plc., 2004.

4. Hickey AJ. Pharmaceutical Inhalation Aerosol Technology. New York, USA: Marcel Dekker, 2004.

5. Current best practice for nebuliser treatment. The Nebulizer Project Group of the British Thoracic Society Standards of Care Committee. Thorax 1997;52 Suppl 2:S1-3.

6. Sukumaran T, Pawankar R, Ouseph J. Asthma diagnosis and treatment - 1009. A clinical study of NE-C900 (OMRON) nebulizer. World Allergy Organ J 2013;6 Suppl 1:P9.

7. Mao L. Formulation considerations for inhaled products. Available online: http://www.catalent.com/index.php/ thinking/science/formulation-of-inhaled-products. Accessed date: 03/08/2017. 2011.

8. Sou T, Meeusen EN, de Veer M, et al. New developments in dry powder pulmonary vaccine delivery. Trends Biotechnol 2011;29:191-8.

9. Chen S, Goode AE, Sweeney S, et al. Sulfidation of silver nanowires inside human alveolar epithelial cells: a potential detoxification mechanism. Nanoscale 2013;5:9839-47.

10. Esper RC, Estrada IE, de la Torre Leon T, et al. Treatment of diffuse alveolar hemorrhage secondary to lupus erythematosus with recombinant activated factor VII administered with a jet nebulizer. J Intensive Care 2014;2:47.

11. Lai SK, Wang YY, Hanes J. Mucus-penetrating nanoparticles for drug and gene delivery to mucosal tissues. Adv Drug Deliv Rev 2009;61:158-71.

12. Harris JM, Chess RB. Effect of pegylation on pharmaceuticals. Nat Rev Drug Discov 2003;2:214-21.

13. Farber MK, Angelo TE, Castells M, et al. Anesthetic management of a patient with an allergy to propylene glycol and parabens. Anesth Analg 2010;110:839-42.

14. O'Riordan TG. Formulations and nebulizer performance. Respir Care 2002;47:1305-12; discussion 1312-3.
15. Pilcer G, Amighi K. Formulation strategy and use of excipients in pulmonary drug delivery. Int J Pharm 2010;392:1-19.

16. Martin AR, Finlay WH. Nebulizers for drug delivery to the lungs. Expert Opin Drug Deliv 2015;12:889-900.

17. Chan JG, Traini D, Chan HK, et al. Delivery of high solubility polyols by vibrating mesh nebulizer to enhance mucociliary clearance. J Aerosol Med Pulm Drug Deliv 2012;25:297-305.

18. Ibrahim M, Verma R, Garcia-Contreras L. Inhalation drug delivery devices: technology update. Med Devices (Auckl) 2015;8:131-9.

19. Heathcote KL, Cockcroft DW, Fladeland DA, et al. Normal expiratory flow rate and lung volumes in patients with combined emphysema and interstitial lung disease: a case series and literature review. Can Respir J 2011;18:e73-6.

20. Hou SG, Wu JY, Li X, et al. Practical, regulatory and clinical considerations for development of inhalation drug products. Asian Journal of Pharmaceutical Sciences 2015;10:490-500.

21. Dennis JH. Chapter 3: New developments in nebulizer technology. In: Boe J, O'Driscoll BR, Dennis JH. editors. Practical Handbook of nebulizer therapy. Ed. CRC Press, Taylor \& Francis Group plc., 2004.

22. Dalby RN, Eicher J, Zierenberg B. Development of Respimat((R)) Soft Mist Inhaler and its clinical utility in respiratory disorders. Med Devices (Auckl) 2011;4:145-55.

23. Fischer A, Stegemann J, Scheuch G, et al. Novel devices for individualized controlled inhalation can optimize aerosol therapy in efficacy, patient care and power of clinical trials. Eur J Med Res 2009;14:71-7.

24. Scheuch G, Siekmeier R. Novel approaches to enhance pulmonary delivery of proteins and peptides. J Physiol Pharmacol 2007;58 Suppl 5:615-25.

25. Ryan G, Singh M, Dwan K. Inhaled antibiotics for longterm therapy in cystic fibrosis. Cochrane Database Syst Rev 2011;(3):CD001021.

26. Maselli DJ, Keyt H, Restrepo MI. Inhaled Antibiotic Therapy in Chronic Respiratory Diseases. Int J Mol Sci 2017;18(5). pii: E1062.

27. Godet C, Cateau E, Rammaert B, et al. Nebulized Liposomal Amphotericin B for Treatment of Pulmonary Infection Caused by Hormographiella aspergillata: Case Report and Literature Review. Mycopathologia 2017;182:709-13.

28. Mantero M, Gramegna A, Pizzamiglio G, et al. Once daily aerosolised tobramycin in adult patients with cystic fibrosis 
in the management of Pseudomonas aeruginosa chronic infection. Multidiscip Respir Med 2017;12:2.

29. Rello J, Sole-Lleonart C, Rouby JJ, et al. Use of nebulized antimicrobials for the treatment of respiratory infections in invasively mechanically ventilated adults: a position paper from the European Society of Clinical Microbiology and Infectious Diseases. Clin Microbiol Infect 2017;23:629-39.

30. Solé-Lleonart C, Roberts JA, Chastre J, et al. Global survey on nebulization of antimicrobial agents in mechanically ventilated patients: a call for international guidelines. Clin Microbiol Infect 2016;22:359-64.

31. USA Clinical trials. Available online: https://clinicaltrials. gov/. Accessed date: 03/08/2017.

32. Fernandez-Garcia R, de Pablo E, Ballesteros MP, et al. Unmet clinical needs in the treatment of systemic fungal infections: The role of amphotericin B and drug targeting. Int J Pharm 2017;525:139-48.

33. Torrado JJ, Serrano DR, Uchegbu IF. The oral delivery of amphotericin B. Ther Deliv 2013;4:9-12.

34. Chan JG, Wong J, Zhou QT, et al. Advances in device and formulation technologies for pulmonary drug delivery. AAPS PharmSciTech 2014;15:882-97.

35. Kuzmov A, Minko T. Nanotechnology approaches for inhalation treatment of lung diseases. J Control Release 2015;219:500-18.

36. Tewes F, Brillault J, Lamy B, et al. Ciprofloxacin-Loaded Inorganic-Organic Composite Microparticles To Treat Bacterial Lung Infection. Mol Pharm 2016;13:100-12.

37. Tewes F, Gobbo OL, Ehrhardt C, et al. Amorphous Calcium Carbonate Based-Microparticles for Peptide Pulmonary Delivery. ACS Appl Mater Interfaces 2016;8:1164-75.

38. Dudley MN, Loutit J, Griffith DC. Aerosol antibiotics: considerations in pharmacological and clinical evaluation. Curr Opin Biotechnol 2008;19:637-43.

39. Anton N, Jakhmola A, Vandamme TF. Trojan microparticles for drug delivery. Pharmaceutics 2012;4:1-25.

40. Thote AJ, Gupta R. Formation of nanoparticles of a hydrophilic drug using supercritical carbon dioxide and microencapsulation for sustained release. Nanomedicine 2005;1:85-90.

41. Tewes F, Ehrhardt C, Healy AM. Superparamagnetic iron oxide nanoparticles (SPIONs)-loaded Trojan microparticles for targeted aerosol delivery to the lung. Eur J Pharm Biopharm 2014;86:98-104.

42. Paranjpe M, Muller-Goymann CC. Nanoparticlemediated pulmonary drug delivery: a review. Int J Mol Sci
2014;15:5852-73.

43. Menon JU, Ravikumar P, Pise A, et al. Polymeric nanoparticles for pulmonary protein and DNA delivery. Acta Biomater 2014;10:2643-52.

44. Yang R, Yang SG, Shim WS, et al. Lung-specific delivery of paclitaxel by chitosan-modified PLGA nanoparticles via transient formation of microaggregates. J Pharm Sci 2009;98:970-84.

45. Rampino A, Borgogna M, Blasi P, et al. Chitosan nanoparticles: preparation, size evolution and stability. Int J Pharm 2013;455:219-28.

46. Gill KK, Nazzal S, JKaddoumi A. Paclitaxel loaded PEG(5000)-DSPE micelles as pulmonary delivery platform: formulation characterization, tissue distribution, plasma pharmacokinetics, and toxicological evaluation. Eur J Pharm Biopharm 2011;79:276-84.

47. Varshosaz J, Ghaffari S, Mirshojaei SF, et al. Biodistribution of amikacin solid lipid nanoparticles after pulmonary delivery. Biomed Res Int 2013;2013:136859.

48. Beck-Broichsitter M, Ruppert C, Schmehl T, et al. Biophysical investigation of pulmonary surfactant surface properties upon contact with polymeric nanoparticles in vitro. Nanomedicine 2011;7:341-50.

49. Nassimi M, Schleh C, Lauenstein HD, et al. Low cytotoxicity of solid lipid nanoparticles in in vitro and ex vivo lung models. Inhal Toxicol 2009;21 Suppl 1:104-9.

50. Gaspar MM, Bakowsky U, Ehrhardt C. Inhaled liposomes-Current strategies and future challenges. J Biomed Nanotechnol 2008;4:245-57.

51. Cipolla D, Gonda I, Chan HK. Liposomal formulations for inhalation. Ther Deliv 2013;4:1047-72.

52. Hood ED, Chorny M, Greineder CF, et al. Endothelial targeting of nanocarriers loaded with antioxidant enzymes for protection against vascular oxidative stress and inflammation. Biomaterials 2014;35:3708-15.

53. EMA. Guideline on the requirements for clinical documentation for orally inhaled products (OIP) including the requirements for demonstration of therapeutic equivalence between two inhaled products for use in the treatment of asthma and chronic obstructive pulmonary disease (copd) in adults and for use in the treatment of asthma in children and adolescents. Available online: http://www.ema.europa.eu/docs/en_GB/document_ library/Scientific_guideline/2009/09/WC500003504.pdf. Accessed date: 01/08/2017. 2009.

54. FDA.Inhaled prorducts. Bioequevalence. Draft Guidance on Fluticasone Propionate; Salmeterol Xinafoate. Available online: https://www.fda.gov/downloads/drugs/guidancec 
omplianceregulatoryinformation/guidances/ucm 367643 . pdf. Accessed date: 01/08/2017. 2013.

55. FDA. Salbutamol sulphate guidelines for bioequivalence products. Available online: https://www.fda.gov/ downloads/Drugs/GuidanceComplianceRegulatoryInf ormation/Guidances/UCM346985.pdf. Accessed date: 01/08/2017. 2013.

56. Solé-Lleonart C, Rouby JJ, Blot S, et al. Nebulization of Antiinfective Agents in Invasively Mechanically

Cite this article as: de Pablo E, Fernández-García R, Ballesteros MP, Torrado JJ, Serrano DR. Nebulised antibiotherapy: conventional versus nanotechnology-based approaches, is targeting at a nano scale a difficult subject? Ann Transl Med 2017;5(22):448. doi: 10.21037/atm.2017.09.17
Ventilated Adults: A Systematic Review and Meta-analysis. Anesthesiology 2017;126:890-908.

57. Ran A. Practical Inhalation Aerosol Technology. 1 ed. Chapel Hill, USA: University of North California, 2004.

58. Schwaiblmair M, Behr W, Haeckel T, et al. Drug induced interstitial lung disease. Open Respir Med J 2012;6:63-74.

59. Yonemori K, Hirakawa A, Kawachi A, et al. Drug induced interstitial lung disease in oncology phase I trials. Cancer Sci 2016;107:1830-6. 